\title{
The Use of Telemedicine in Pulmonary Conditions
}

\author{
Martini $\mathrm{N}^{1}$, Panthappattu $\mathrm{J}^{2}$, Verma $\mathrm{S}^{1,3,4}$ and Khanijo $\mathrm{S}^{1 *}$ \\ ${ }^{1}$ Touro College of Osteopathic Medicine, USA \\ ${ }^{2}$ St. George's University School of Medicine, Grenada, West Indies \\ ${ }^{3}$ Department of Pulmonary, Critical Care and Sleep Medicine, Northwell Health, USA \\ ${ }^{4}$ York College of The City University of New York, USA
}

\section{Review Article}

Volume 3 Issue 4

Received Date: July 11, 2020

Published Date: July 30, 2020

DOI: $10.23880 /$ jqhe-16000173

*Corresponding author: Sameer Khanijo, M.D, F.C.C.P, North well Health, Pulmonary, Critical

Care and Sleep Medicine, 410 Lakeville Rd., Suite 107, New Hyde Park, NY 11040, Tel: (516) 465-5400; Email: SKhanijo2@ northwell.edu

\section{Abstract}

Telemedicine is the use of digital technology that, remotely, allows patients to receive healthcare services from their providers. It is cost efficient, easily accessible and can improve access to medical care among patients in rural communities. However, the growth of telemedicine has been slow due to skepticism and privacy concerns. Nevertheless, the recent outbreak of COVID-19 has brought telemedicine into the limelight. A Medline/PubMED search was performed to find articles with "telemedicine" as a heading.

Keywords: Telemedicine; Pulmonary Hypertension; COVID-19; Pulmonary Rehabilitation; Ultrasound

\section{Introduction}

The field of telemedicine has been around for nearly half a century, but it is only over the past few decades that telemedicine has changed drastically. As technology advances, the field of telemedicine is poised to evolve and impact patient care by finding its way into mainstream medical practice. Recently, the emergence of the COVID-19 pandemic has drastically changed the way we provide medical care and has brought telemedicine to the forefront.

The World Health Organization defines telemedicine as "the delivery of health care services, where distance is a critical factor, by all health care professionals using information and communication technologies, for the exchange of valid information for diagnosis, treatment, and prevention of disease and injuries, research and evaluation, and for the continuing education of health care providers, in all the interests of advancing the health of individuals and their communities" [1]. In other words, it is the use of communications technology to provide healthcare resources to those in urgent need.
Telemedicine is currently implemented in various branches of medicine, such as neurology and radiology. The use of telemedicine in diagnosing and treating stroke has been one of the most successful applications of this technology, earning it the title "telestroke" [2]. According to the American Heart Association, "the goal of telestroke medicine is to promptly provide an immediate stroke expertdirected clinical assessment, a review of tests, a diagnosis, and an emergency management plan" [2].

One of the most immediate benefits of telestroke medicine is the ability to rapidly administer treatment. Surprisingly, its use is limited. In a study of the utilization of tPA in the treatment of acute ischemic stroke by hospitals in the US, it was determined that $64 \%$ of hospitals did not use tPA to treat any patients during the totality of the twoyear study [3]. It is possible that such poor utilization of treatment could be due to the lack of stroke expertise within hospitals, especially within smaller or rural communities [3]. Telestroke offers these hospitals the benefits of having an onsite specialist without the actual physical presence of one. 
The benefits of telemedicine have been demonstrated across different medical specialties. These advantages are largely a consequence of the convenience and low cost of its implementation [1,4]. Patients living in remote areas are often unable to travel long distances to visit their physicians, especially for minor ailments. Smartphones and videoconferencing offers an alternative for both patients and clinicians to "modify" their approach to care. In turn, this change can consequently lead to a lower volume of emergency room visits and doctor's office visits. Beyond convenience and low cost, telemedicine also empowers patients with chronic conditions [5]. Patients with complex medication regimens may have difficulty managing their medications. Under these circumstances, the use of any digital health platform can streamline treatment plans and enhance patient medication adherence.

It is clear that telemedicine will continue to expand, but there is still some uncertainty about its use. As such, it is not yet universally accepted. The risk of data security breach is an important consideration that may prevent patients and physicians from sharing vital information. Another limitation is the possibility of unintentional sharing of information to a third party source. Liability risks are also high with telemedicine because the quality of care provided during a telemedicine consultation might be inferior to that one would receive in a doctor's office. This can have an impact on reaching a proper diagnosis [4]. Because of more reliance on telemedicine, there also remains a justified concern about healthcare providers losing their jobs.

\section{Telemedicine, Ultrasound and e-ICU}

There is a known shortage of critical care intensivists in the US, complicating the process of patient evaluation and decision-making in the ICU settings. Using teleintensivists, facilities lacking critical care physicians are able to incorporate specialists, showing them real-time ultrasound images to aid in the diagnosis and treatment of critically ill patients [6]. Tele-guided ultrasound has shown a $96 \%$ success rate in a pilot study where a provider with minimal ultrasound training could obtain images at bedside and review them, in real time, with a tele-intensivist [7]. The tele-physicians could effectively instruct ultrasound users at bedside in order to obtain the necessary images. This application of tele-ultrasound may be useful not only in the ICU setting, but also in the ambulatory and rural settings. By providing access to e-ICU and tele-intensivists, communities lacking in these services can more effectively pursue point of care management options [6].

\section{Telemedicine and Pulmonary Conditions}

As previously mentioned, many studies have evaluated the use of telemedicine in stroke management. Telemedicine has also shown efficacy in the management of other diseases such as diabetes, congestive heart failure and chronic liver disease, with similarly reduced costs and improved convenience. Among pulmonary conditions, telemedicine is showing potential benefits in the management of chronic obstructive pulmonary disease (COPD) and asthma, as well as in pulmonary rehabilitation. These benefits include home-based clinician diagnosis and monitoring, as well as data interpretation at dedicated centers [8]. Knowing that patients with COPD may experience disease exacerbation at some time, telemedicine can be used to monitor for signs of exacerbation, potentially decreasing the frequency of hospital admissions and associated healthcare expenses [8].

\section{Telemedicine and Pulmonary Rehabilitation}

A sub-division of telemedicine referred as telerehabilitation is an important staple of therapy in the management of COPD. Intra-hospital pulmonary rehabilitation facilities are scarce. Tele-rehabilitation can be used to supplement face-to-face rehabilitation centers. After a COPD exacerbation, patients can continue with their exercise regimen at home following discharge instead of coming to specialized rehabilitation centers. Providers can use video conferencing, phone calls, emails and internet software as tools to ensure medication adherence, assess condition, monitor and possibly intervene [9].

\section{Telemedicine and Pulmonary Hypertension}

Pulmonary hypertension (PH) is a rare and devastating condition that leads to progressively increasing shortness of breath and poor outcomes. With no cure, $\mathrm{PH}$ is best managed with medications that improve quality of life. As previously discussed, telemedicine is rapidly evolving into a new staple of standard therapy in COPD and asthma management. However, to date there are no telemedicine services available for PH patients [10]. It is fair to extrapolate the benefits of telemedicine that are observed in COPD and asthma patients to those with $\mathrm{PH}$. These include assessing patient status after hospital discharge, ensuring medication compliance and encouraging compliance with CPAP and oxygen.

\section{Telemedicine and COVID-19}

The COVID-19 pandemic created a disruption among social infrastructures, including the healthcare system. Telemedicine was making positive impact prior to the pandemic, yet some members of the medical community were skeptical of its utility and were not willing to embrace it. The social distancing polices and lockdown orders due to COVID-19 have forced the medical community to reassess the utility of telemedicine. A recent survey of oncologists 
demonstrated that despite a high degree of awareness $(82 \%)$ of telemedicine and its potential application, only $59 \%$ of oncologists were implementing it in their practice [11]. Nevertheless, of the doctors surveyed, 46\% responded 'definitely' when asked if they preferred to virtually manage their patients [11].

As the pandemic continues, more data is coming to light. Preliminary data from a study conducted at an HIV prevention clinic in Rio de Janeiro showed that telemedicine is bridging the gap between physicians and patients in terms of the delivery of pre-exposure prophylaxis (PrEP) [12]. By implementing telemedicine in the clinics, physicians have been able to provide patients with rapid HIV testing, teleconsults, and long-term supplies of PrEP [12]. By eliminating lapses in coverage, patients have been able to avoid medication shortages and reduce the time needed to pick up their PrEP refills [12].

In response to the pandemic, the United States experienced a seismic shift amongst various medical organizations and governmental policies with statements being issued endorsing telemedicine as a means of delivering healthcare, as well as guidelines for its usage. Healthcare systems needed to amend their practices to allow continuity of care, while simultaneously minimizing the risk of COVID-19 infection amongst medical staff and other patients. The result has been an aggressive incorporation of virtual conduits, such as video conference via smartphone, by which healthcare workers are able to communicate with their patients. By using these modalities, early prescreening can be performed for any patient who possibly shows symptoms of COVID-19, as well as for follow-up of asymptomatic patients.

As health systems and medical practices gear up for virtual links, providers may need some form of training before employing this technology. Additionally, they may also need to address privacy concerns that may jeopardize a patient's information. Many providers are also concerned about continuity of care amongst non-english speaking patients and those with limited internet connectivity. While the latter two concerns may take some time to resolve, it is possible to secure information technology domains and provide expedited training to health care providers and staff. This training could be similar to the training received by new interns and residents in using a hospital's electronic medical record.

While there are certainly challenges in using telemedicine that were confirmed by its use during the pandemic, we have seen increased implementation and usage across various domains and the ease of access to care that it has provided patients as a result.

\section{Conclusion}

Telemedicine has proven itself in a variety of specialties to be an accessible and cost-effective means of delivering healthcare. It is an exclusive industry that continues to grow exponentially. While there are many benefits to using telemedicine, its limitations cannot be ignored. Therefore, governments and various health agencies should continue to be involved in its development to improve quality of care to patients. Given its slow rise and current trajectory, it is unclear what the future of telemedicine holds. However, the current COVID-19 pandemic has made telemedicine an indispensable tool in the delivery of modern day healthcare. More large scales studies are needed to fully understand the health outcomes of telemedicine.

\section{Conflict of Interest: None}

\section{Financial Disclosure: None}

\section{References}

1. Organization WH (2010) Telemedicine: opportunities and developments in Member States: report on the second global survey on eHealth. World Health Organization.

2. Wechsler LR, Demaerschalk BM, Schwamm LH, Adeoye OM, Audebert HJ, et al. (2017) Telemedicine Quality and Outcomes in Stroke: A Scientific Statement for Healthcare Professionals From the American Heart Association/ American Stroke Association. Stroke 48: e3-e25.

3. Kleindorfer D, Xu Y, Moomaw CJ, Khatri P, Adeoye O, et al. (2009) US geographic distribution of rt-PA utilization by hospital for acute ischemic stroke. Stroke 40: 35803584.

4. Alvandi M (2017) Telemedicine and its Role in Revolutionizing Healthcare Delivery. The American Journal of Accountable Care 5: e1-e5.

5. Pare G, Jaana M, Sicotte C (2007) Systematic review of home telemonitoring for chronic diseases: the evidence base. J Am Med Inform Assoc 14: 269-277.

6. Zaidi G, Koenig S (2016) Merging Two Worlds: Point-ofCare Ultrasonography and Tele-Medicine. Chest 149(2): 605-606.

7. Levine AR, McCurdy MT, Zubrow MT, Papali A, Mallemat HA, et al. (2015) Tele-intensivists can instruct nonphysicians to acquire high-quality ultrasound images. J Crit Care 30: 871-875. 
8. Cooper CB (2009) Respiratory applications of telemedicine. Thorax 64: 189-191.

9. Ambrosino N, Vagheggini G, Mazzoleni S, Vitacca M (2016) Telemedicine in chronic obstructive pulmonary disease. Breathe (Sheff) 12(4): 350-356.

10. Hernandez C, Mallow J, Narsavage GL (2014) Delivering telemedicine interventions in chronic respiratory disease. Breathe (Sheff) 10: 198-212.
11. Tashkandi E, Zeeneldin A, AlAbdulwahab A, Elemem O, Elsamany, et al. (2020) Virtual Management of Patients With Cancer During the COVID-19 Pandemic: Web-Based Questionnaire Study. J Med Internet Res 22: e19691.

12. Hoagland B, Torres TS, Bezerra DRB, Geraldo K, Pimenta C, et al. (2020) Telemedicine as a tool for PrEP delivery during the COVID-19 pandemic in a large HIV prevention service in Rio de Janeiro-Brazil. Braz J Infect Dis. 